\section{RETORNO AL CAMPAMENTO: RESISTENCIA Y MELANCOLÍA EN LOS MÁRGENES DE LA CIUDAD FORMAL*}

\author{
Rodrigo Esteban Morales Martínez ${ }^{\alpha}$ \\ Carolina Beatriz Besoain Arrau ${ }^{\beta}$ \\ Alejandro Soto Morales ${ }^{\gamma}$ \\ Laís Pinto de Carvalho ${ }^{\delta}$ \\ Karla Dominique Hidalgo Pino ${ }^{\varepsilon}$ \\ Ismael Fernández Posada ${ }^{\zeta}$ \\ Vicente Bernal Santibáñez ${ }^{\eta}$
}

\section{Resumen}

Este artículo expondrá los principales resultados de una investigación cuyo objetivo fue explorar la experiencia que algunos pobladores han desarrollado en relación con la política habitacional chilena. Siguiendo la pista a hallazgos anteriores, se discutirá los procesos de subjetivación de pobladores/ as que, habiendo recibido la vivienda social entre las décadas de 1980 y 2000, decidieron regresar al campamento. Desde lógicas de la investigación social cualitativa y del método etnográfico, se realizaron múltiples técnicas de producción de datos, entre 2014 y 2015, en dos campamentos de las comunas de Lampa y San Bernardo, en Santiago. Los

\section{BACK TO THE SETTLEMENT: RESISTANCE AND MELANCHOLY ON THE BORDERS OF THE FORMAL CITY*}

\author{
Rodrigo Esteban Morales Martínez ${ }^{\alpha}$ \\ Carolina Beatriz Besoain Arrau ${ }^{\beta}$ \\ Alejandro Soto Morales ${ }^{\gamma}$ \\ Laís Pinto de Carvalho ${ }^{\delta}$ \\ Karla Dominique Hidalgo Pino ${ }^{\varepsilon}$ \\ Ismael Fernández Posada ${ }^{\zeta}$ \\ Vicente Bernal Santibáñez ${ }^{\eta}$
}

\section{Abstract}

This paper presents the main findings of research whose objective was to explore the experience of some residents within the Chilean housing policy context. Based on previous studies, this research analyzes the processes of subjectivisation experienced by a group of residents who, despite being provided with social housing from 1980 to 2000, decided to return to their former settlements. Qualitative social research and ethnographic methods were used to compile data for two settlements located in the municipalities of Lampa and San Bernardo, Santiago, for the period 2014 to 2015. This data was analyzed 
datos fueron analizados desde propuestas del análisis del discurso. Los resultados muestran el retorno al campamento como un contra-movimiento que resiste a los procesos de subjetivación gatillados por el tránsito hacia la vivienda social. Una resistencia que detiene el proceso orientándose hacia un pasado idealizado, en el que se añora otra experiencia de reconocimiento y habitar. Revisaremos la noción de retorno en su doble sentido, de resistencia y fracaso, movilizado por la búsqueda de un lugar imposible que condensa deseos, frustraciones y conflictos de la experiencia de habitar la ciudad desde los márgenes de su formalidad.

\section{PALABRAS CLAVE: POLITICA DE VIVIENDA, CAMPAMENTOS, SUBJETIVIDAD, RESISTENCIAS POPULARES, SEGREGACIÓN.}

\section{Recibido: 29/04/2016}

Aceptado: 10/03/2017

Investigación financiada por el Fondo "Fomento a Proyectos de Investigación/Facultad de Psicología", Universidad Alberto Hurtado del año 2014.

a Chile. Profesor colaborador Universidad de Chile y Universidad Alberto Hurtado. Correo electrónico: rodrigo.morales.mz@gmail.com

$\beta \quad$ Chile. Profesora auxiliar, Facultad de Psicología, Universidad Alberto Hurtado. Correo electrónico: cbesoain@uahurtado.cl

Chile. Estudiante de Magister Universidad Alberto Hurtado. Profesor colaborador Universidad de Chile. Correo electrónico: ps.alejandro.soto@gmail.com

Chile. Profesora colaboradora Universidad Andrés Bello. Correo electrónico: Icarvalho@uc.cl

Chile. Licenciada en Psicología, Universidad Alberto Hurtado. Correo electrónico: kahidalg@gmail.com

Chile. Licenciado en Psicología, Universidad Alberto Hurtado. Correo electrónico: ismaelfernandezposada@gmail.com

Chile. Licenciado en Psicología, Universidad Alberto Hurtado. Correo electrónico: vichobernal@gmail.com from a perspective of discourse analysis. It is suggested that the repopulation of settlements is a counter-movement to subjectivation processes generated by the provision of social housing, the result being the disruption of the housing process, the idealization of the past and the yearning for different recognition and inhabiting experiences. This paper analyzes the concept of return in the two senses of resistance and failure determined by the search for an impossible place that reflects the different wishes, frustrations and conflicts common to the experience of inhabiting the borders of the formal city.

\section{KEYWORDS: HOUSING POLICY, SETTLEMENTS, SUBJECTIVITY, POPULAR RESISTANCE, SEGREGATION.}

Received: 29/04/2016

Accepted: 10/03/2017

\footnotetext{
This research was financed by the "Fund for the Promotion of Research Projects/ Faculty of Psychology" project, Alberto Hurtado University, 2014.

$\alpha \quad$ Chile. Professor, University of Chile and Alberto Hurtado University. Email: rodrigo. morales.mz@gmail.com

$\beta \quad$ Chile. Professor Faculty of Psychology, Alberto Hurtado University. Email: cbesoain@uahurtado.cl

$\gamma \quad$ Chile. Ms student Alberto Hurtado University. Professor, University of Chile. Email: ps.alejandro.soto@gmail.com

$\delta \quad$ Chile. Professor, Andres Bello University. Email: Icarvalho@uc.cl

Chile.Psychology graduate, Alberto Hurtado University. Email: kahidalg@gmail.com Chile. Psychology graduate, Alberto Hurtado University. Email: ismaelfernandezposada@gmail.com

$\eta \quad$ Chile. Psychology graduate. Alberto Hurtado University. Email: vichobernal@gmail. com
} 


\section{INTRODUCCIÓN}

Los procesos de modernización que ha experimentado Chile en las últimas décadas han ido cambiando diferentes ámbitos de la vida social y cultural. Tanto las ciudades como sus habitantes han experimentado transformaciones producto del despliegue de un proyecto económico basado fundamentalmente en la liberalización del mercado y la disminución del rol garante del Estado en asuntos económicos. Estos procesos, instalados forzosamente durante la dictadura militar y profundizados durante el retorno a la democracia, han generado una tensa convivencia entre el acelerado desarrollo económico y las enormes desigualdades en la distribución de la riqueza. Efecto de ello es el creciente malestar social y subjetivo que denuncia, con poco éxito, la esquiva traducción del progreso en experiencias de bienestar para todos los ciudadanos y no solo para algunos (PNUD, 2012; Mayol y Ahumada, 2015).

Durante las últimas décadas, el supuesto de la modernización chilena ha encontrado su sostén discursivo tanto en el tono trascendental e ilustrado otorgado a la economía de mercado chilena como en el aumento del acceso a bienes de consumo por parte de la población. Sin embargo, a pesar de su contundencia performativa, ambos supuestos revelan enormes contradicciones y simplismos de lo que significa un proceso de modernización (Mayol y Ahumada, 2015). Y es que el incremento desigual de ingresos salariales, las libertades crediticias de los bancos, la dominancia de bienes importados a bajo costo, junto a otros dinamismos de la economía de mercado han generado, más que un proceso de modernización, una acusada precarización y cortoplacismo en un crecimiento de tipo capitalista dependiente y rentista (Mayol y Ahumada, 2015).

Al entender un proceso de modernización como un tipo de crecimiento económico sustentado en procesos de diversificación productiva (incremento y generación de nuevos sectores) y sus correlatos en la modificación de la estructura social (cambios en la composición laboral) resulta comprensible que, ante la hipótesis de la modernización, Mayol y Ahumada (2015, p. 27) refieran el caso chileno como un "crecimiento sin modernización". Y es que para ellos la hipótesis de la modernización no es sino una falacia discursiva, conducente de una imagen país distorsionada en la relación economía, política y sociedad. Por otra parte, para Garretón (2007) esta apariencia "ilustrada y trascendental", al referirse al progreso del país, formaría parte de aquel "mito" del éxito y la ejemplaridad del "modelo chileno", sostenido convenientemente por diversos actores económicos internacionales atraídos por las facilidades comerciales del proyecto chileno. 
En este sentido, las nuevas metáforas en términos de movilidad -la desterritorialización, el desplazamiento, la migración- nos ha hecho más conscientes del hecho de que la dinámica principal de la cultura y la economía ha sido alterada significativamente. La experiencia de lugar, de territorio, ha sufrido transformaciones que precisan de nuevas comprensiones para las nuevas geometrías de poder (Massey, 2005) y sus consecuencias en consonancia con un examen crítico de esta hipótesis modernizadora (Escobar, 2000; Giddens, 2003; Touraine, 1994).

Ahora bien, los impactos de esta modalidad chilena de modernización se encuentran en directa relación con las políticas sociales implementadas en las últimas décadas. Estas últimas, entendidas como aquellas formas de intervención social, han tenido por objetivo realizar un tipo de justicia social y económica siempre en concordancia con el modelo de desarrollo definido por el mismo Estado (Corvalán, 1996). De este modo, la política social ha tenido desde su inicio una relación contradictoria con el neoliberalismo, puesto que aquello que la caracteriza es la regulación de la actividad privada y la asignación de recursos por vías independientes a las del mercado. Así, la política social viene operando en gran medida como un sistema correctivo a los efectos de tal dinámica mercantil, estando sujeta, por ende, a sus reglas y vicisitudes.
En este escenario las políticas sociales habitacionales han sido un importante mecanismo para conseguir el supuesto ideal de modernización de las ciudades. La ciudad de Santiago, en particular, ha experimentado un crecimiento exponencial desde principios de siglo XX producto de las migraciones campo ciudad. Así, los asentamientos informales o tomas de terreno han sido, durante décadas, el modo mediante el cual los migrantes "sin techo" han ido resolviendo sus necesidades habitacionales, llegando a transformarse durante las últimas décadas en una relevante fuerza social y política (Garcés, 2002).

El Estado y la sociedad civil respondieron de diversos modos a las demandas habitacionales y de participación de los pobladores. Los últimos treinta años, tras la liberalización del suelo urbano y la creación del subsidio habitacional, la respuesta se ha caracterizado por un desplazamiento de las antiguas responsabilidades del Estado al individuo, transformando la vivienda en un bien al que se accede con esfuerzo y ahorro familiar (Hidalgo, 2005; Güell, 1999), en un movimiento que Sisto (2009) ha caracterizado como transformación ideológica de la cuestión social.

Desde el regreso a la democracia, la tasa de construcción de viviendas sociales aumentó de un modo exponencial. La inversión del sector público en el campo de la vivienda social fue cuantiosa, 
realizándose un aumento del 53\% en materia habitacional en esos años (Hidalgo, 2005). Tal como sugiere Skewes (2005), el éxodo masivo desde asentamientos irregulares a viviendas sociales tomó la forma de una verdadera transición entre mundos de vida, un tránsito hacia la modernidad. Un tránsito, sin embargo, atravesado por importantes problemáticas - principalmente referidas a las consecuencias malignas de la segregación residencial y la mala calidad de la construcciónque comenzaron a ser denunciadas desde los años noventa (Ducci, 1997; Hidalgo, 2005; Rodríguez y Sugranyes, 2004; Sabatini, Cáceres y Cerda, 2001).

Esta investigación se propuso estudiar el impacto de las políticas habitacionales subsidiarias en la experiencia de aquellos pobladores que han experimentado estos tránsitos. En particular se exploraron las tensiones entre los procesos de subjetivación que la política de vivienda chilena instala en sus beneficiarios (Besoain, 2012; Besoain y Cornejo, 2015) y cierta resistencia, encarnada en el movimiento de retorno al campamento que algunos pobladores han desarrollado en relación con aquellos procesos. Siguiendo la pista a trabajos previos (Skewes, 2005; Márquez, 2003 y 2004; Besoain, 2012; Besoain y Cornejo, 2015) la presente investigación exploró los procesos que participan de la experiencia de pobladores que, habiendo sido beneficiados por alguno de los tipos de solución habitacional que han sido implementados por el Estado de Chile, decidieron retornar al campamento.

El estudio de la experiencia del retorno al campamento radica tanto en la relevancia que la comprensión de este fenómeno tiene para la actual política de vivienda chilena, como por el potencial político que este movimiento sintetiza en relación con los procesos de subjetivación dominantes en la escena chilena contemporánea. Comprenderlo es relevante pues se trata de un movimiento que pone en tensión las aspiraciones de la política social en una trayectoria silenciosa e invisibilizada, que desafía la legalidad y el sentido común relativo al habitar. No se tiene claridad de sus dimensiones cuantitativas, quedando además por fuera de los márgenes del discurso político institucional. Asimismo, es un fenómeno que anida desde sus pequeños nichos el malestar y la resistencia a la forma de vida propuesta por las políticas de vivienda.

Desde una perspectiva crítica, postulamos la relevancia de hacer visible la relación entre las transformaciones económicas, sociales y materiales chilenas en la constitución subjetiva de sus ciudadanos, a modo de poner de frente el impacto del modelo de desarrollo imperante. Así, desde el entendimiento de la psicología como una práctica sociocultural, que se constituye y es constituyente de un entramado político (Parker, 1996; Morales, 
2010 y 2013), nos parece ineludible prestar atención a dichos efectos, desde una mirada que rescate y discuta críticamente tanto sus condicionantes como sus consecuencias.

A continuación presentaremos algunos antecedentes teóricos y empíricos que constituyeron el marco conceptual desde el cual nos aproximamos al fenómeno del retorno al campamento. Esto es, (1) la relación entre procesos de subjetivación y prácticas discursivas, (2) la noción de "heterotopía" para la comprensión del campamento y su relación con la ciudad formal, (3) las dinámicas de apropiación y apego a lugar y (4) los antecedentes de añoranza del campamento y la fantasía del retorno de investigaciones previas.

\section{Discurso, poder y procesos de subjetivación}

Sabemos que en toda sociedad la producción del discurso se encuentra controlada, seleccionada y redistribuida por una serie de procedimientos que tienen por función invocar ciertos poderes, dominar el acontecimiento cotidiano al mismo tiempo que desarrollar estrategias con el fin de esquivar las insurgencias ante el proyecto discursivo dominante (Foucault, 1999). Tales procedimientos de control implican por ello importantes estrategias de exclusión y la consecuente escisión entre lo verdadero/bueno y lo falso/malo, imponiendo así una gestión del saber que establece un nivel de competencia y un marco de condiciones que donan legitimidad al discurso.

Esta operatoria diseminada y abierta del poder, articula así lo que entenderemos como procesos de subjetivación: la constitución relacional de un tipo de sujeto -un cuerpo y sus relaciones posiblesque, mediante diversas operaciones productivas, se reconoce y replica legitimando de este modo las prácticas discursivas que lo constituyen. Estas prácticas discursivas, o prácticas de verdad, son propias de una forma que toma la cultura en un tiempo determinado y establecen la diferencia entre lo que puede ser dicho y lo que no, delimitando así un particular orden del discurso. La operatoria de estos procesos de subjetivación implicará sin embargo la trascendencia al puro discurso capturando directamente al cuerpo y sus potencialidades (como sujeto o población). Por ello, tanto en su gestión individual como grupal encontraremos operaciones que pueden ir desde la administración estadística de la población (una biopolítica) hasta la preocupación por las gestualidades más elementales del cuerpo (una microfísica del poder).

En este sentido, al transitar desde una descripción del sujeto hasta los procesos de subjetivación que lo condicionan y legitiman podemos encontrarnos con un despliegue discursivo y estético de 
normalizaciones recursivas. Ahora bien, tal recursividad tiene la particularidad de haber transitado durante las últimas décadas desde un sistema de normalización centrado en el control heteronormativo a un sistema de coerción autónomo, que no opera como una estructura vigilante sino que se internaliza como una autovigilancia orientada a la capitalización de uno mismo. En este sentido, Han (2014) distingue el presente neoliberal como un espacio donde, a diferencia de la sociedad del castigo o de la disciplina vigilante, vivimos a partir de una sensación opuesta: la de no ser sujetos sometidos y de vivir por el contrario en un proyecto libre en constante reinvención de sus posibilidades. No hay por ello la sensación de dominación soberana ni delimitación del espacio, sino la autovigilancia respecto de la capitalización de uno mismo como infraestructura en el curso de las demandas de la modernización (Han, 2014, p. 25).

Así, para Han (2014), la liberación como proyecto del yo en el escenario actual no es sino la proyección de una fantasía en el desplazamiento desde coacciones externas y coerciones ajenas a coacciones internas y coerciones propias donde el objetivo de ambas será ya no la obediencia al amo soberano, ni el control disciplinar, sino que tomará su forma como coacción al rendimiento y optimización de la conducta, donde amo y esclavo coincidirán ahora en el mismo lugar: el cuerpo y psique del individuo (Han, 2014).

\section{Campamento como heterotopía}

Para la comprensión del movimiento del "retorno", resultará útil la noción de "heterotopía" acuñada por Michel Foucault (1997). Éste al respecto comprende las heterotopías como espacios o lugares efectivos que existen en toda cultura y que han sido diseñados en la institucionalidad misma de esas sociedades. La particularidad de estos espacios es que operan como "contra emplazamientos" que representan simbólica y materialmente todos los demás espacios de esa cultura. Así estos contra emplazamientos, estos "lugares otros", no representan simplemente una síntesis de la organización social de una comunidad sino que despliegan lo que podríamos entender como el inconsciente o la sombra de la cultura que los constituye. Por ello se presentan invirtiendo los lugares que representan, o cuestionándolos, revelan en su conjunto aspectos de la cultura que son reprimidos y en cierta forma eyectados de la misma, pero que dada su relevancia, deben ser localizables.

Los espacios de marginalidad -tomas de terreno, campamentos- operarán así como una particular densidad heterotópica cuya función al sistema en su totalidad parece ser un recordatorio tópico de los espacios legítimos y así de las buenas y malas formas de vida que favorecen o no -según el uso 
que se le dé al cuerpo, al espacio y al tiempo- la posibilidad de crecimiento en tanto movilidad social. Esto último, en el ya señalado proceso de modernización, no será si no la ubicación y la movilidad en territorios que promuevan el acceso a mayores capacidades de consumo y endeudamiento.

El campamento, la toma de terreno, no es entonces "otro espacio" entre muchos, sino más bien aquel "espacio otro", cuya función no es accidental para la reproducción del sistema: este espacio marginal es un límite material de la ciudad formal, con lo cual tenemos que la materialidad informal del campamento o de la toma de terreno, su tiempo informal, su organización informal, resultan marginales en tanto operan como reflejo funcional para la ciudad y sus formalidades en el proceso de modernización. Tales lugares heterotópicos finalmente no producen nada por si mismos en tanto su densidad marginal es producto ya de la ciudad formal.

Esto genera que lugares como tomas de terreno o campamentos informales $-\mathrm{y}$ sus particulares relaciones con el tiempo, la economía, las añoranzas, entre otros- operen estableciendo, desde su ejercicio del límite una profunda diferencia entre las representaciones legítimas de la cultura versus aquellas marginadas, excluidas; no obstante, incluidas en tanto marginalidades localizables.

\section{Apropiación y apego al lugar}

La relación que establecen las personas con los lugares que habitan es subjetiva y material, construida a partir de una adaptación mutua, donde el espacio influye en la persona tanto como esta lo transforma de acuerdo a sus deseos y posibilidades. Por ello, el habitar no es tan solo tener un lugar donde vivir, sino que está íntimamente ligado al concepto de construir (Heidegger, 1994), el cual es entendido como edificar un contexto, un entorno de vida que responde a las necesidades, modos de ser y aspiraciones de las personas: es un espacio que se crea y recrea constantemente (Chardon, 2010, p. 22).

La experiencia de habitar es influida por los distintos ámbitos de la vida, tales como el espacio físico, las relaciones sociales y alternativas culturales, las posibilidades económicas o el clima emocional (Blunt \& Dowling, 2006). Todo aquello se pone en juego en la capacidad de los seres humanos para apropiarse de los lugares y hacerlos parte fundamental de su experiencia. Por ello, la apropiación es entendida por Vidal y Pol (2005) como un mecanismo básico de las personas, el cual permite la interiorización de la experiencia a través de los significados que le otorgamos a la realidad. Es un proceso dialéctico por el cual se relacionan las personas y los espacios en un contexto sociocultural 
que comprende personas, grupos, comunidades y la sociedad.

Ahora bien, este mecanismo se produce a través de dos vías complementarias (Pol, 1996; Vidal y Pol, 2005); la acción-transformación y la identificación simbólica. La primera da cuenta del modo en el que las personas actúan sobre los entornos, las personas y los grupos dejando señales, o huellas marcadas simbólicamente, generando a la vez que estas señales se interioricen e incorporen en los procesos cognitivos y afectivos. Las personas transforman los espacios y les otorgan significados individuales y sociales en la interacción. A su vez, a través de la identificación los individuos se reconocen en el entorno, auto atribuyendo las cualidades de este como parte de su propia identidad.

Este proceso dialéctico entre la acción-transformación y la identificación simbólica será mediado por las condiciones de habitabilidad que se presenten y del contexto sociocultural en el que se esté inmerso (Low y Altman, 1992). Desde acá, el modo en el que sea gestionado el tránsito hacia la vivien$\mathrm{da}$, las relaciones que se establezcan con el lugar de origen, las expectativas puestas sobre el lugar de destino, las características materiales y sociales de la nueva vivienda, afectarán las posibilidades de su apropiación.
El proceso de apropiación es un proceso imprescindible de considerar en toda política habitacional. Los hallazgos de Manzo (2014) muestran que habitantes beneficiados por políticas de vivienda social efectivamente pueden desarrollar relaciones de apego a sus unidades, blocks y a la comunidad de la cual son parte. Para ello es clave el sentido de estabilidad, las redes de vecinos y el involucramiento en un proyecto común (Manzo, 2014). La autora también propone que el apego de estos habitantes es dinámico, e incluye una dimensión negativa, conformando un apego ambivalente. Son emociones complejas y conflictivas expresadas en una contradicción entre el arraigo y el deseo de vivir en otro lugar. El vínculo de las personas con los lugares se desarrolla y cambia al largo del tiempo (Manzo, 2003), siendo el tiempo de residencia uno de los clásicos predictores positivos del apego al lugar (Kleit y Manzo, 2006; Lewicka, 2011).

Sin embargo, en Chile, los hallazgos de Rodríguez y Sugranyes (2004) revelan que el aprecio por la vivienda social va decreciendo con el pasar de los años de residencia, apareciendo el desencanto entre los seis meses y los dos años de instalados. En sus estudios, el 64,5\% de los habitantes encuestados manifestó querer irse de la vivienda. La mayoría de sus motivos eran de índole social (mala convivencia entre vecinos, percepción de inseguridad, 
delincuencia y drogadicción) y en segundo lugar fueron reportados dimensiones físicas de las viviendas, el aislamiento y la falta de servicios.

\section{Añoranza y fantasía de retorno al campamento}

En congruencia con los procesos de subjetivación señalados y la resistencia ante los mismos, además de la función social del campamento como heterotopía de la ciudad formal y las dinámicas de apego y apropiación al lugar, surge una dimensión mucho más íntima en los pobladores que se despliega como añoranza y fantasía de retorno desde la vivienda social al campamento.

Esta añoranza y/o fantasía constituye una dimensión muy relevante en la experiencia de los pobladores que recibieron su vivienda social. Investigaciones previas (Besoain, 2012; Besoain y Cornejo, 2015) muestran esta fantasía como expresión de una añoranza por los modos de la sociabilidad y la subjetividad propios de la vida en el campamento. En algunos casos esta fantasía se convirtió en realidad, dando lugar a un tránsito inverso a los esfuerzos de formalización e inclusión social que la política social chilena ha venido desarrollando. Este fenómeno ha sido motivo de preocupación para la política de vivienda y ha participado de la dinámica interna de las tomas de terreno desde sus comienzos. Habitualmente este fracaso de la política pública se ha atribuido a la dificultad de los pobladores para pagar las nuevas cuentas y deudas que la vivienda social significa, lo que motivó que la primera administración de Michelle Bachelet (2006-2010) realizara ajustes en la Política de Vivienda como la implementación de la vivienda dinámica sin deuda y los planes de habilitación social:

El gran cambio propuesto por la política de vivienda vigente, que atiende a los sectores más carenciados, elimina el crédito asumiendo que las familias cuentan con poca capacidad de endeudamiento. A pesar de esta limitación, se supone que al no existir una obligación de pago se liberan recursos para ser empleados en la terminación de la vivienda. El concepto es el llamado Vivienda Social Dinámica sin Deuda y es aplicable a las viviendas entregadas mediante el Fondo Solidario de Vivienda, explicado más adelante. La estructura de financiamiento considera 10 UF de ahorro, más 280 a 320 UF de Subsidio Directo, lo que entrega un valor de 290 a 330 UF disponibles para el sitio, la urbanización y construcción de la vivienda. El crédito eliminado, se suple por un aumento del monto de subsidio respecto de lo que había antiguamente para las viviendas del mismo estrato. Al mismo tiempo se reduce la superficie de vivienda entregada a una unidad inicial de unos $32 \mathrm{~m}^{2}$, que debe contemplar su futura ampliación para alcanzar una superficie 
edificada de a lo menos $50 \mathrm{~m}^{2}$ (Aguirre, D'Alençon, Justiniano y Faverio, 2006, p. 3).

A pesar de algunos esfuerzos concretos de la política pública ha sido muy difícil la erradicación definitiva de estos asentamientos informales. El catastro realizado por el Ministerio de Vivienda y Urbanismo (s.f.) el año 2011 reveló que aun existían 657 campamentos a nivel nacional (167 campamentos más que en el año 2007) y 27.378 familias viviendo en estas condiciones. El año 2013 la Fundación TECHO realizó una actualización del catastro del 2011 la que reveló que habría 925 campamentos a nivel nacional -268 más de los catastrados por el Ministerio el año 2011- (TECHO, 2013). Las cifras y su crecimiento a lo largo de los años revelan la actualidad y relevancia de este fenómeno a pesar de los amplios esfuerzos que se han desplegado desde la política pública desde el regreso a la democracia.

La añoranza por el campamento o la fantasía por retornar al mismo, si bien, no cuentan con datos estadísticos, aparecen cualitativamente como un fenómeno característico en pobladores que han sido beneficiarios de la vivienda social dinámica sin deuda (Besoain y Cornejo, 2015). Esta añoranza es vivida como malestar el cual, sin embargo, es permanentemente regulado desde narrativas de "maternidad sacrificial". Es decir, cada vez que aparece la añoranza por el retorno esta es regulada con algún enunciado correctivo que itera en los beneficios que la nueva vivienda podrá implicar para los propios hijos. A pesar de la regulación, este malestar igualmente consigue tensionar la celebración de la nueva intimidad y la sensación de libertad y autonomía conseguida con la nueva vivienda. Y es que tal libertad y autonomía se restringe fundamentalmente a detalles estéticos de la vida cotidiana que no conseguían traducirse en un proyecto de reconocimiento y orientado hacia un futuro, quedando los pobladores constreñidos en una temporalidad presentista. La añoranza tomaba también la forma de prácticas anacrónicas e imágenes que desafiaban los modos del habitar propios de la vivienda social, haciendo visible prácticas y modos de habitar propias de otro tiempo, así como narrativas de derecho y de lucha política colectiva, que décadas atrás habían sido articuladoras del movimiento de pobladores de Santiago (Garcés, 2002).

\section{Método}

Esta investigación se ha situado bajo las lógicas de la investigación social cualitativa (Cottet, 2006), asumiendo la palabra como evento dialógico y contextual, donde se articula el sentido y el significado (Cornejo, Besoain y Mendoza, 2011). Desde estas premisas se construyó un diseño de carácter exploratorio y comprensivo, inspirado en las propuestas de perspectivas etnográficas (Hammersley y Atkinson, 1994). Es así como se diseñó un 
dispositivo de producción de información compuesto por una variedad de técnicas -entrevista, grupo de discusión, observación participante, fotografías, recorridos, cuadernos de campo- reunidas para conseguir una aproximación multifacética y reflexiva del fenómeno del retorno al campamento.

\section{PARTICIPANTES}

Los participantes de esta investigación fueron pobladores que habiendo recibido algún tipo de solución habitacional a través de la política de vivienda la dejaron, vendieron o abandonaron. Hoy viven en campamentos en la Región Metropolitana de Chile y no tienen opción de entrar en la política habitacional, ya que el subsidio se otorga por una sola vez al beneficiario.

Se realizó un muestreo intencionado a través de informantes clave, así como del procedimiento de "bola de nieve" (Patton, 2002). Los informantes clave fueron ex-participantes de la investigación doctoral de una de las investigadoras. El número final de participantes fueron siete pobladores -seis mujeres y un varón- de dos campamentos ubicados en las comunas de San Bernardo y Lampa de la ciudad de Santiago, con diversidad de trayectorias habitacionales. Dos de ellos fueron usuarios de la política habitacional entre al año 2000 y 2005. Los demás fueron usuarios en la década de los '80. Las modalidades fueron diversas (Ministerio de
Vivienda y Urbanismo, 2004) (a) caseta sanitaria: edificación compuesta por baño, cocina y/o lavadero, que cuenta con servicios de agua potable y sistema de evacuación de aguas servidas; (b) vivienda pública construida originalmente para familias de miembros de las fuerzas armadas, pero que fueron destinadas luego a familias sin techo durante la dictadura militar; y (c) vivienda social dinámica sin deuda: vivienda flexible, dinámica y modular, de menor tamaño y que puede ser completada en el tiempo por los usuarios.

\section{LOS TERRITORIOS DE LAMPA Y SAN BERNARDO}

Las comunas están ubicadas en la Región Metropolitana (RM) de Santiago de Chile. Lampa en la zona rural norponiente, en la Provincia de Chacabuco. San Bernardo en la zona sur, en la Provincia de Maipo.

Según datos del Ministerio de Desarrollo Social (2014a y 2014b), ambas comunas presentan una tasa de pobreza superior al nivel regional y nacional, así como una proporción mayor de hogares cuyas viviendas están en condiciones de hacinamiento medio o crítico, contando con un importante déficit habitacional. Las comunas se caracterizan por su composición rural y urbana. Especialmente en Lampa, donde la mayoría de su población es de origen campesino, ligados a actividades agrícolas. San Bernardo, si bien, también presenta una 
TABLA 1. CARACTERIZACIÓN DE PARTICIPANTES SEGÚN TRAYECTORIA HABITACIONAL

\begin{tabular}{|c|c|c|c|c|c|c|c|}
\hline $\begin{array}{l}\text { Participante } \\
\text { seudónimo }\end{array}$ & $\begin{array}{l}\text { Lugar de } \\
\text { origen }\end{array}$ & $\begin{array}{l}\text { Tipo de } \\
\text { vivienda } \\
\text { recibida }\end{array}$ & $\begin{array}{l}\text { Lugar y } \\
\text { año de } \\
\text { recepción de } \\
\text { la vivienda } \\
\text { social }\end{array}$ & $\begin{array}{l}\text { Tiempo en } \\
\text { vivienda } \\
\text { social } \\
\text { (aprox.) }\end{array}$ & $\begin{array}{l}\text { Tipo de } \\
\text { salida de la } \\
\text { vivienda }\end{array}$ & $\begin{array}{l}\text { Razón principal } \\
\text { de la salida }\end{array}$ & $\begin{array}{l}\text { Lugar de } \\
\text { vivienda } \\
\text { actual }\end{array}$ \\
\hline Beatriz & $\begin{array}{l}\text { Campamento en } \\
\text { San Bernardo. }\end{array}$ & $\begin{array}{l}\text { Vivienda diná- } \\
\text { mica sin deuda. }\end{array}$ & $\begin{array}{l}\text { San Bernardo, } \\
\text { año } 2000 .\end{array}$ & 1 año. & Abandono. & $\begin{array}{l}\text { Malas costumbres } \\
\text { de los vecinos, no } \\
\text { reconocerse en el } \\
\text { lugar, busca otro } \\
\text { ambiente para sus } \\
\text { hijos. }\end{array}$ & $\begin{array}{l}\text { Campamento en } \\
\text { San Bernardo. }\end{array}$ \\
\hline Sandra & $\begin{array}{l}\text { Campamento en } \\
\text { San Bernardo. }\end{array}$ & $\begin{array}{l}\text { Vivienda diná- } \\
\text { mica sin deuda. }\end{array}$ & $\begin{array}{l}\text { San Bernardo, } \\
\text { año } 2000 .\end{array}$ & $\begin{array}{l}1 \text { año y } 6 \\
\text { meses. }\end{array}$ & Abandono. & $\begin{array}{l}\text { Desilusión de los } \\
\text { vecinos, añoranza } \\
\text { por vida en el } \\
\text { campamento. }\end{array}$ & $\begin{array}{l}\text { Campamento en } \\
\text { San Bernardo. }\end{array}$ \\
\hline Verónica & $\begin{array}{l}\text { Campamento en } \\
\text { Lampa. }\end{array}$ & $\begin{array}{l}\text { Vivienda } \\
\text { militar. }\end{array}$ & $\begin{array}{l}\text { La Pintana, } \\
1984 .\end{array}$ & 6 meses. & Abandono. & $\begin{array}{l}\text { Delincuencia y } \\
\text { violencia militar en } \\
\text { el sector. }\end{array}$ & $\begin{array}{l}\text { Campamento en } \\
\text { Lampa. }\end{array}$ \\
\hline Paulina & $\begin{array}{l}\text { Allegada en } \\
\text { Lampa. }\end{array}$ & $\begin{array}{l}\text { Vivienda diná- } \\
\text { mica sin deuda. }\end{array}$ & Colina, 1997. & 1 año. & $\begin{array}{l}\text { Abandono } \\
\text { Se quedó su } \\
\text { ex-marido vi- } \\
\text { viendo ahí. Hoy } \\
\text { recibe un pe- } \\
\text { queño arriendo } \\
\text { informal. }\end{array}$ & $\begin{array}{l}\text { Violencia, tanto } \\
\text { intrafamiliar como } \\
\text { asociada a la cer- } \\
\text { canía a la cárcel de } \\
\text { Colina. }\end{array}$ & $\begin{array}{l}\text { Campamento en } \\
\text { Lampa. }\end{array}$ \\
\hline
\end{tabular}




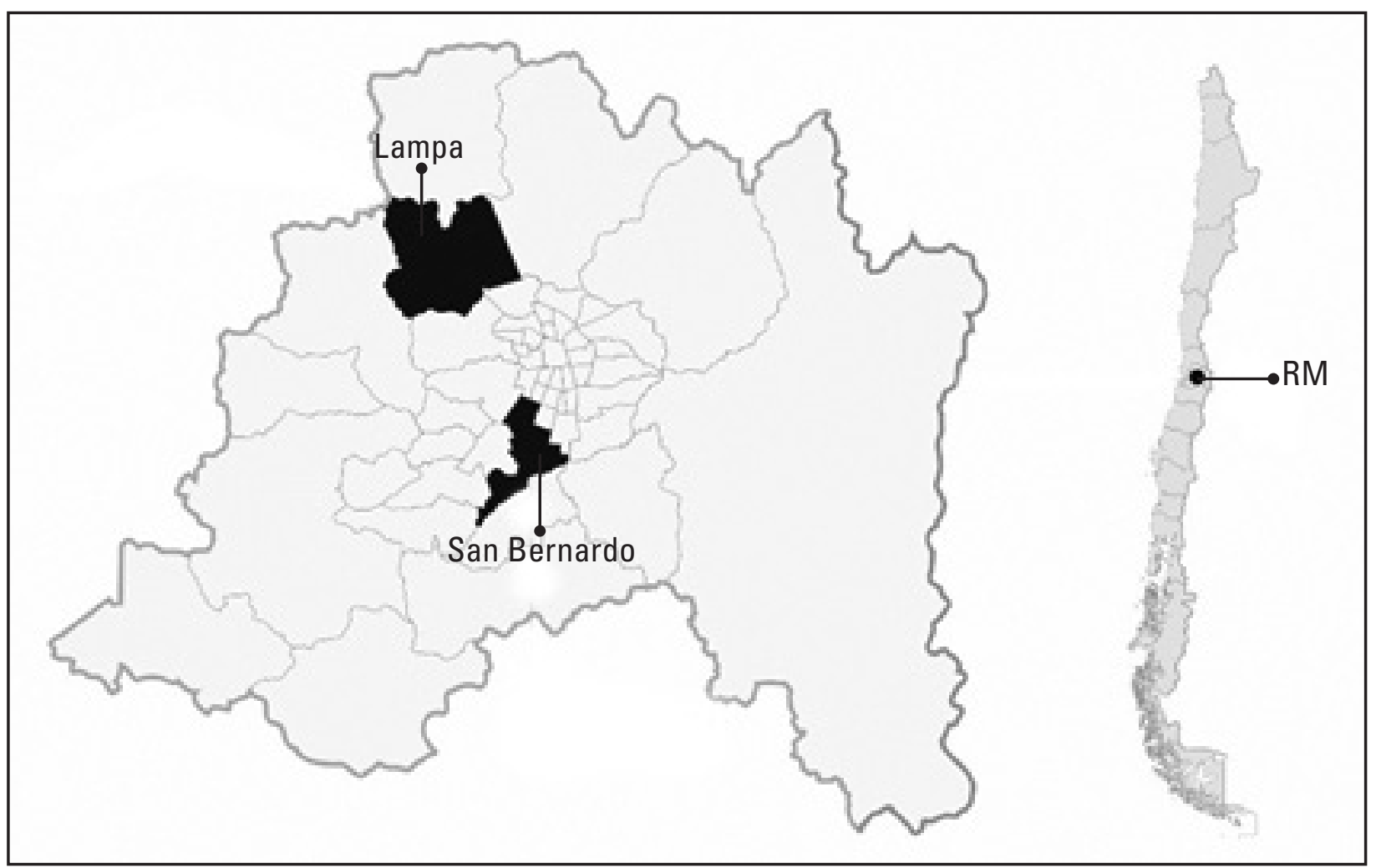


composición rural, esta es menor. Su accesibilidad involucra grandes avenidas, carreteras y tres estaciones de metrotren.

\section{TÉCNICAS DE PRODUCCIÓN DE DATOS}

Se realizaron tres entrevistas narrativas individuales (Cornejo, 2006) y una entrevista grupal a la que asistieron tres pobladoras (Cottet, 2006). Además se realizaron dos jornadas de observación participante (Hammersley y Atkinson, 1994) para observar las dinámicas y modos de vida de los pobladores, tanto en los campamentos como en uno de los barrios de vivienda social de proveniencia durante el transcurso. Se trabajó con fotografías de las viviendas, espacios comunes y prácticas sociales de los pobladores, tanto en los campamentos como en uno de los barrios de vivienda social.

\section{ANÁLISIS DE DATOS}

Se realizó un análisis guiado por las propuestas del análisis de discurso. El foco estuvo puesto en los discursos utilizados para hacer sentido de la propia experiencia y cómo estos producen identidades, actividades y relaciones (Starks y Brown, 2007). Siguiendo a Arfuch (2002), se buscó desarrollar una escucha plural poniendo énfasis en el acontecimiento del decir y la producción dialógica del sentido.
El análisis se sostuvo en la realización de diversos dispositivos de escucha (Cornejo, Besoain y Mendoza, 2011). Uno de ellos fue los cuadernos reflexivos del investigador, que acompañaron tanto las entrevistas individuales, la entrevista grupal como las observaciones participantes. También durante la fase de análisis de datos se realizaron reuniones de interanálisis y jornadas con expertos, las que contribuyen a dar mayor densidad y rigor a la construcción de resultados.

\section{CONSIDERACIONES ÉTICAS}

Para resguardar la voluntariedad de participación se utilizaron documentos de consentimiento informado con cada uno de los participantes, los que culminaron con la lectura y firma de los mismos antes de iniciar la producción de los datos. Los datos fueron producidos entre los meses de octubre 2014 y junio 2015. Para resguardar el anonimato de los participantes se ha decidido usar seudónimos y modificar la información que pudiera hacerlos reconocibles.

\section{Resultados}

A continuación presentaremos algunas reflexiones en torno a los principales hallazgos de esta investigación, deteniéndonos en tres dimensiones que nos 
parecen relevantes para comprender la experiencia del retorno al campamento y el proceso de subjetivación que está implicado en dicho movimiento. Estas son, (1) el retorno como interrupción de un habitar intolerable, (2) resistencia, fracaso y culpa, y por último (3) retorno y melancolía.

\section{RETORNO COMO INTERRUPCIÓN DE UN HABITAR INTOLERABLE}

"¿Por qué? Porque te golpeaban la puerta, que te probaban llaves, que la delincuencia estaba abajo, no podías bajar, no podías salir a buscar trabajo. Yo tampoco salia, si mis chiquillos quedaban solos. Entonces, decidí dejarlo tirado y juro por Dios Santo, que no me ha pesado. No me va a pesar nunca haberlo dejado. ¿Qué ahí que tendría ahora? Pura delincuencia". (Entrevista grupal, Lampa).

La pregunta por el retorno implica introducirse en la comprensión de un contra-movimiento. Es decir, se trata de un movimiento que interrumpe otro movimiento - preparado, anticipado y muchas veces deseado- hacia un lugar que contenía la promesa de convertirse en hogar, fuente de reconocimiento, espacio de resguardo y pertenencia a una comunidad: la ciudad formal. En tanto interrupción este movimiento se inscribe como trayectoria antagónica al proceso de subjetivación promovido por la política pública. Desde el Estado aparece como signo de una falla, en principio incomprensible, frente a la cual el retorno queda instalado en el territorio de lo marginal, lo raro, e incluso lo delictual.

Los resultados de esta investigación muestran sin embargo que el retorno es, a veces, la única alternativa frente a un presente insoportable. Se instala en las tramas de una salida desesperada, de una huida inevitable frente a un enemigo que amenaza con la destrucción de aquello que se erige como la fuente de sentido y de valor más primordial en ese sujeto. El momento en el que el poblador realiza el retorno, si bien pudo haberse deseado o fantaseado previamente, aparece como un momento desesperado, como el único camino para la sobrevivencia de lo amado -a veces un hijo en riesgo de volverse drogadicto-, pero también ante el riesgo de perderse a sí mismo, de transformarse en alguien a quien ya no es posible reconocer. En este sentido hay en el retorno un movimiento hacia la preservación, hacia el cuidado de una continuidad del sí mismo, en tanto no es posible apropiarse, apegarse y reconocerse en aquel lugar.

"Bueno, yo de allá me vine por el trabajo de mi esposo, no habia trabajo. Las deudas se iban juntando. Y ya se había empezado a cambiar el ambiente, empezó a llegar la droga. Mi hijo era adolescente, yo tenía que trabajar, mi marido trabajaba, entonces él estaba tomando malas juntas y malas costumbres. $Y$ antes de que mi hijo se me echara a perder, yo no la pensé dos veces, vendí y me vine". (Entrevista grupal, Lampa). 
La experiencia del habitar, entendida como una construcción subjetiva entre las personas y los lugares, a través de las actividades cotidianas y las relaciones con los otros, se vuelve hostil, impidiendo que las personas sientan como propio el espacio y puedan proyectarse en él (Chardon, 2010; Ortiz, Vieyra y Orozco, 2015). Los sentimientos asociados a vivir en la vivienda social son de extrañeza y de miedo ante el entorno.

En el discurso de "los retornados" los enemigos en la vivienda social son diversos según la época: en dictadura el miedo era a la violencia militar, mientras en democracia las principales figuras son la delincuencia y la droga. Pero para todos el barrio y el espacio público aparecen como lugares que no son posibles de intervenir ni de transformar y tampoco de investir con la propia subjetividad, por lo tanto, la única posibilidad es la huida para alejarse de los otros y sus amenazas. Es así como para quienes retornan, el proceso de hacer vínculos con el entorno y de desempeñar un papel en el barrio y con los vecinos no se logra, no pudiéndose construir un sentido de pertenencia en que los individuos se reconozcan y sean reconocidos a través de la participación y el desarrollo de la comunidad (Sanín, 2008).

\section{RESISTENCIA FRACASO Y CULPA}

"De lo que siento yo así como una derrota, fue el haber perdido mi casa. Como ustedes saben, por lo menos lo que es el subsidio, tú puedes postular una sola vez. Yo por lo menos ya no lo voy a volver a poder hacer". (Entrevista Verónica, Lampa).

En tanto resistencia el retorno es un movimiento subjetivo hacia la diferenciación de aquella alteridad que se aparece como destino en la vivienda social. El retorno es desde acá un movimiento de reconocimiento por negatividad: "yo no soy ese". En tanto fracaso el retorno es experimentado como la frustración de un proyecto que se hizo imposible de sostener. Un fracaso que es vivido como falta de recursos personales y familiares para dar continuidad a una vida demasiado exigente. El retornado aparece para sí mismo como quien ha fracasado en tanto no ha sido capaz de soportar las demandas y amenazas de la ciudad formal. Luego, tal como lo veíamos al inicio de este trabajo, el que retorna en este sentido no puede volcar sino sobre sí mismo la experiencia de esta derrota.

Tras el retorno, sin embargo, el campamento no consigue sentirse como el territorio que se añoraba. Esto es lo que fragua la resistencia con la sensación de fracaso, de cansancio, de derrota. Y es que la resistencia que motivó el retorno implica 
un gran esfuerzo que cuesta sostener en el tiempo. Así, en la experiencia de habitar el campamento luego del retorno, se oscila entre el deseo de volver a tener la oportunidad de poseer una propiedad y el resguardo de aquello que se protege como sagrado: un modo de habitar que se reconoce como propio y trascendente a todo lugar.

Esto podemos observarlo, por ejemplo, en la resistencia de los pobladores entrevistados a reconocerse en primera instancia como habitantes de campamento; es decir, a configurar desde ese referente nominal su identidad como sujetos. Por el contrario, en vez de la figura del campamento como delimitación del reconocimiento aparecen caracterizaciones en las que ellos se distinguen antes: son gente de trabajo, a las que no les han regalado las cosas, que pagan sus cuentas, tienen buenas costumbres y educan a sus hijos. Es decir, se describen por sus acciones más que por la heterotopía que parecen encarnar. Así el reconocimiento opera desde la diferenciación de esa alteridad denostada, esos otros, que no son ellos, o que pueden aparecer como ellos solo cuando son ubicados ahí por las cooptaciones dominantes de la cultura que los sitúa en ese lugar y que en tal colocación los reduce a mero dato estadístico.

"Yo me vine de allá, porque de cierta manera las casas eran muy lindas y todo, pero la gente lo empezó a transformar en tipo campamento. Era una villa linda, y la misma gente la destruyó. Tenían otras costumbres y el entorno no era bueno, había mucha delincuencia. Bueno, la verdad es que yo no me acostumbré a eso. Yo por eso retorné de nuevo a mi casa. No quería que mis hijos crecieran ahi”. (Entrevista Beatriz, San Bernardo).

Del mismo modo aparece vinculado al retorno una resistencia ligada a la concepción de la buena vida y la lucha por esta. La vida en calma, el respeto, la seguridad, la comunidad son cuestiones que se sienten perdidas en el tránsito a la vivienda social. El retorno aparece como resistencia a las nuevas formas de vida. Se retorna porque no se encuentra el respeto, la calma, la seguridad, el adecuado uso de los espacios, incluso la higiene. Aparece así una falla relacional que impide la sensación de pertenecer a una comunidad.

Los pobladores se sienten en una lucha que van perdiendo, lo cual promueve vivencias entre nostalgia y desesperación. Se sienten indefensos y vulnerados al no ser escuchados, reconocidos, legitimados en su deseo de hogar. Sin embargo, a pesar de que perciben la entrega de una nueva vivienda como un derecho y una necesidad de todas las personas, en su caso particular la dificultad de obtener una casa -o del reconocimiento de un hogar en marcha- se ha individualizado e internalizado como un problema propio y por una falla propia, es por esto que priman la nostalgia y la sensación de culpabilidad. Luego entonces, esta falla si bien inicialmente se traduce en un repliegue hacia la 
vida privada del hogar, finalmente como movimiento resulta insuficiente, culminando en el retorno - este segundo movimiento- que si bien evidencia el fracaso de la comunidad, termina siendo vivido como fracaso en primera persona.

\section{RETORNO Y MELANCOLÍA}

"Bueno, yo ya después cuando volví, retorné a mi antigua casa, ya no era mi antigua casa. Ya no había casas, las expropiaron todas y nos quedamos solamente los de este lado, que es el lado de puros adultos mayores nomás. Igual hubo un cambio". (Entrevista Sandra, San Bernardo).

El movimiento de retorno se instala entre dos topos: el aquí y el allá. En los relatos, estas marcas discursivas hacen nacer dos territorios, dos espacios diferentes, a momentos opuestos, heterotopías decíamos al inicio de este artículo. Sin embargo, ambas marcas cargan un sentido que permite dibujar cierto paisaje común para el allá que señala el destino del retorno, inscrito en tramas de un lugar original que se añora y que es motor del deseo actual de movimiento. El allá que es el destino del retorno se inscribe en el registro de un territorio extraviado, en el que habita cierta promesa: se trata de una tierra que parece conocerse, pero cuya coincidencia con el lugar en el que se vivió alguna vez resulta borrosa.
Tras dejar el campamento, y ante lo insoportable de la vida en la vivienda social, este parece transfigurarse, para convertirse en un espacio excepcional, el cual se recuerda como una comunidad unida, sin conflictos, de personas que pueden reconocerse miembros de un mismo nosotros. Así el retorno es un movimiento de regreso, material y a la vez imaginario, a un espacio añorado tanto como fantaseado, en el que residiría la promesa de la buena vida.

La precariedad material y las razones que antes motivaron una salida -también deseada- no aparecen en esta parte del relato, quedan obturadas para el deseo del retorno. El campamento se recrea como un lugar idealizado, haciéndose casi invisible el malestar asociado a dicha precariedad. Es una insistencia a momentos romántica, a momentos trágica, que configura aquel lugar como cobijo, en la imagen de la casa inolvidable (Bachelard, 2000).

De este modo es posible afirmar que el lugar del retorno es un lugar atópico, un "sin lugar", donde el movimiento por volver revela un esfuerzo por restablecer una continuidad con lo que se fue. En otras palabras, puede ser pensado como un movimiento para reivindicar la memoria de quien se ha sido en una escena donde la permanencia en la vivienda social amenaza con sepultar ese pasado y 
ese sujeto. Sin embargo, no es del todo posible ese restablecimiento, ni la posibilidad de hacer hogar nuevamente: están presentes la desilusión de que el lugar ya no es lo que se recordaba, las transformaciones relacionales que han vivido y el sentimiento de caída de la comunidad.

También, en un registro más tangible, la ilegalidad de estos asentamientos hace correr el riesgo constante de ser reubicados o derechamente expropiados, lo cual genera una sensación de incertidumbre e inestabilidad cotidiana. Esto último dificulta a su vez el establecimiento de relaciones profundas y colaborativas con los vecinos, lo que conlleva más bien percepciones de lejanía e incluso hostilidad.

“¿Pero qué saco con arreglar más, si me va a pasar lo mismo? Entonces mientras no tenga la posibilidad de tener un pedacito de terreno donde me caiga mi casa, donde yo pueda construir, donde yo pueda a empezar a hacer algo donde yo diga 'ya, esto lo hago y esto es definitivo"'. (Entrevista grupal, Lampa).

La capacidad de apropiación e identificación con este espacio se ve así interrumpida por el temor a perderlo todo nuevamente, lo que genera a la vez la dificultad para intervenir, transformar y proyectarse en este lugar. No hay agencia del espacio, menos aún propiedad, y el estado anímico del poblador no es así otro que el de la melancolía.

\section{Conclusiones}

Los resultados expuestos sugieren que la política de vivienda chilena favorecería una cierta relación de no agenciamiento o apropiación del espacio por parte del poblador sino, a la inversa, un agenciamiento o apropiación del poblador por parte del territorio: es el agenciado y no el agente.

Tanto en la vivienda social como en el campamento al que se retornó, la experiencia de los pobladores es la de un apego ambivalente, por lo cual prima la sensación de inseguridad. El momento del retorno es una resistencia que lucha por recuperar el sentido de apropiación. Sin embargo, luego del retorno, se instala nuevamente la dificultad de apropiación bajo la forma de melancolía por una sensación de pérdida inlocalizable: "estoy donde quiero estar, pero no consigo estar del modo en el que me gustaría estar". Y es que el poblador no retorna a un lugar cualquiera sino, como decíamos antes, a un lugar otro -heterotópico- un contra emplazamiento que cumple una función de espejar el proyecto social de la ciudad formal. Un lugar que se sirve del sujeto como objeto límite de contraste respecto de lo que implican lo ideales de la modernización neoliberal: movilidad social, capacidad de consumo, capacidad de endeudamiento, entre otros. 
Esta melancolía toma lugar luego en las relaciones entre vecinos que se permean de esta incertidumbre: la falta de agencia y el ambivalente apego al lugar conducen un frágil vínculo en las relaciones interpersonales, las cuales tienden a ser más bien funcionales y pocas veces íntimas y confiables. El riesgo de desalojo aumenta la incertidumbre y la sensación de fragilidad: este contra-emplazamiento se vuelve anti-social. Ello conlleva la dificultad de establecer una relación profunda con el entorno, es decir, de apertura a sus múltiples diferencias, de correr el riesgo y el placer del encuentro con el otro, de agenciar finalmente el lugar. Por el contrario, ante tal imposibilidad lo que acontece es un repliegue ambivalente hacia el hogar: "esta es mi casa... la que puedo perder en cualquier momento". En este movimiento se va descomponiendo la posibilidad de comunidad dejando si más al poblador volcado sobre sí mismo.

Es por ello que los resultados nos sugieren que la política habitacional, a pesar de sus esfuerzos por mejorar la calidad de vida de las personas, va coludiendo igualmente con un proceso de retirada de la comunidad, la cual los pobladores suplementan con un repliegue hacia el hogar privado. Lo anterior parece ser un efecto sintomático de la difícil apropiación del espacio: el espacio solamente se entrega, se inaugura, se celebra, mas no hay una dialéctica que sintetice este nuevo reconocerse en el lugar. La comunidad así deviene en tanto espacio a-tópico, precario de sentido y motor de la extrañeza: tanto como ausencia de reconocimiento de sí mismo en el lugar, como añoranza de un lugar donde la comunidad acontecía donando, en gran medida, este sentido ahora ausente.

Así, el proceso de subjetivación del poblador retornado se caracteriza por un retraimiento que hemos llamado melancólico en el sentido de acontecer como búsqueda de un lugar imposible de recuperar, lo que deja al sujeto añorando un modo de habitar que solo existe en el imaginario de un territorio mítico, que alguna vez fue habitado, pero que hoy no logra existir. En su odisea, el poblador que retorna experimenta algunas sensaciones de triunfo, pero generalmente su historia acontece desde el fracaso, predominando la sensación de una culpa personal que socava la otrora fuerza de la comunidad. Así, al no poder con lo que el mandato de la cultura -la ciudad formal-y esos otros ciudadanos parecen si poder, el poblador, en su espacio marginal queda entregado a una vivencia marcada por la melancolía.

Esta investigación visibiliza un fenómeno que debe seguir siendo investigado. En tanto estudio exploratorio, estos resultados tienen un carácter de hipótesis emergentes que deberían seguir siendo profundizadas y discutidas en relación con diversas variables no abordadas por esta investigación. Futuras investigaciones deberían profundizar en diferencias en la experiencia de retorno de acuerdo 
al momento en el que se fue beneficiario de la política de vivienda, si en dictadura, si en los noventa, o luego de la Nueva Política Habitacional hasta hoy vigente. Asimismo, deberían explorarse diferencias en relación con la edad y el género, las cuales tampoco fueron analizadas en este estudio.

\section{Agradecimientos}

Agradecemos a la Facultad de Psicología de la Universidad Alberto Hurtado por el apoyo y confianza brindada. Agradecemos también a los pobladores participantes por permitirnos escuchar sus historias y pensar junto a ellos.

\section{Bibliografía}

Aguirre, C., D'alençon, R., Justiniano, C. y Faverio, F. (2006). Lineamientos generales para la terminación de las viviendas dinámicas sin deuda. Resumen investigación y propuesta. Recuperado de http://www.subdere.gov.cl/sites/default/files/ documentos/articles-69777_recurso_1.pdf

Arfuch, L. (2002). El espacio biográfico. Dilemas de la subjetividad contemporánea. México: Fondo de Cultura Económica.

Bachelard, G. (2000). La poética del espacio. México: Fondo de Cultura Económica.
Besoain, C. (2012). Vivienda social y subjetividades urbanas en Santiago: espacio privado, repliegue presentista y añoranza. (Tesis de Doctorado), Pontificia Universidad Católica de Chile, Santiago. Recuperado de http://dspace2.conicyt. cl/handle/10533/89114

Besoain, C. y Cornejo, M. (2015). Vivienda social y subjetivación urbana en Santiago de Chile: espacio privado, repliegue presentista y añoranza. Psicoperspectivas, 14(2), 16-27. doi:10.5027/PSICOPERSPECTIVAS-VOL14ISSUE2-FULLTEXT-369

Blunt, A. y Dowling, R. (2006). Home. New York: Taylor $\&$ Francis

Chardon, A.-C. (2010). Reasentar un hábitat vulnerable. Teoría versus praxis. Revista INVI, 25(70), $17-$ 75. doi:10.4067/S0718-83582010000300002

Cornejo, M. (2006). El enfoque biográfico: trayectorias, desarrollos teóricos y perspectivas. Psykhe, 15(1), 95-106. doi:10.4067/ S0718-22282006000100008

Cornejo, M., Besoain, C. y Mendoza, F. (2011). Desafíos en la generación de conocimiento en la investigación social cualitativa contemporánea. Forum Qualitative Sozialforschung / Forum: Qualitative Social Research, 12(1).

Corvalán, J. (2006). Los paradigmas de lo social y las concepciones de intervención en la sociedad. Recuperado de http://biblioteca.uahurtado.cl/ UJAH/Reduc/pdf/pdf/7934.pdf 
Cottet, P. (2006). Diseños y estrategias de investigación social. El caso de la ISCUAL. En M. Canales (Ed.), Metodologías de investigación social (pp. 185-216). Santiago: LOM.

Ducci, M. E. (1997). Chile: el lado oscuro de una político de vivienda exitosa. EURE Revista Latinoamericana de Estudios Urbano Regionales, 23(69).

Escobar, A. (2000). El lugar de la naturaleza y la naturaleza del lugar: ¿Globalización o postdesarrollo? En la colonialidad del saber: eurocentrismo y ciencias sociales. Perspectivas Latinoamericanas. Buenos Aires: CLACSO.

Foucault, M. (1997). Los espacios otros. Astrágalo: revista cuatrimestral iberoamericana(7), 83-91.

Foucault, M. (1999). Ética, estética y hermenéutica obras esenciales, volumen III. Barcelona: Paidós.

Garcés, M. (2002). Tomando su sitio: el movimiento de pobladores de Santiago, 1957-1970. Santiago: LOM.

Garretón, M. (2007). Del postpinochetismo a la sociedad democrática. Globalización y política en el Bicentenario. Santiago: Ramdom House Mondadori.

Giddens, A. (2003). Un mundo desbocado. Los efectos de la globalización en nuestras vidas ( $5^{a}$ ed.). Madrid: Taurus.

Güell, P. (1999). Familia y modernización en Chile. Recuperado de http://chitita.uta.cl/cursos/2011-1/0000439/recursos/r-8.pdf

Hammersley, M. y Atkinson, P. (1994). Etnografía: métodos de investigación ( ${ }^{a}$ ed.). Barcelona: Paidós.
Han, B. C. (2014). Psicopolitica. Barcelona: Herder.

Heidegger, M. (1994). Construir, habitar, pensar. En Conferencias y artículos. Barcelona: Serbal.

Hidalgo, R. (2005). La vivienda social en Chile y la construcción del espacio urbano en el Santiago del siglo XX. EURE Revista Latinoamericana de Estudios Urbano Regionales, 31(93), 108-112. doi:10.4067/S0250-71612005009300009

Kleit, R. y Manzo, L. (2006). To move or not to move: Relationships to place and relocation choices in HOPE VI. Housing Policy Debate, 17(2), 271308. doi:10.1080/10511482.2006.9521571

Low, S. y Altman, I. (1992). Place attachment: a conceptual inquiry. En I. Altman y S. Low (Eds.), Place attachment (pp. 1-12). New York: Plenum Press.

Manzo, L. (2003). Beyond house and haven: toward a revisioning of emotional relationships with places. Journal of Environmental Psychology, 23(1), 47-61. doi:10.1016/S0272-4944(02)00074-9

Manzo, L. (2014). Exploring the shadow side. place attachment in the context of stigma, displacement, and social housing. En L. Manzo y P. Devine-Wright (Eds.), Place attachment. advances in theory, methods and applications (pp. 178-190). New York: Routledge.

Márquez, F. (2003). Identidad y fronteras urbanas en Santiago de Chile. Psicologia em revista, 10(14), 35-51. 
Márquez, F. (2004). Márgenes y ceremonial: los pobladores y las políticas de vivienda social en Chile. Politica (43), 185-203.

Massey, D. (2005). La filosofía y la política de la espacialidad: algunas consideraciones. En Pensar este tiempo: espacios, afectos, pertenencias (pp. 101-128). Buenos Aires: Paidós.

Mayol, A. y Ahumada, J. M. (2015). Economía política del fracaso. La falsa modernización del modelo neoliberal. Santiago: Ocho Libros.

Ministerio de Desarrollo Social. (2014a). Reporte comunal Lampa: Región Metropolitana Informes Comunales. Recuperado de http://observatorio. ministeriodesarrollosocial.gob.cl/indicadores/ pdf/comunal_general/metropolitana/Lampa_2013.pdf

Ministerio de Desarrollo Social. (2014b). Reporte comunal San Bernardo: Región Metropolitana Informes Comunales. Recuperado de http:// observatorio.ministeriodesarrollosocial.gob. cl/indicadores/pdf/comunal_general/metropolitana/San_Bernardo_2013.pdf

Ministerio de Vivienda y. Urbanismo. (2004). Chile. Un siglo de politicas en vivienda y barrio. Santiago: Autor.

Ministerio de Vivienda y. Urbanismo. (s.f.). Catastro 2011: mapa social de campamentos. Resultados generales. Santiago: Autor. Recuperado de http://www.minvu.cl/incjs/download. aspx?glb_cod_nodo=20110523144022\&hdd_ nom_archivo $=$ Catastro.pdf.
Morales, R. (2010). Psicoterapia sistémica, micropolítica y subjetividad: alcances en torno a la formación. En Formación en y para una psicología clinica. (pp. 45-67). Santiago: FACSO.

Morales, R. (2013). El desdoblamiento psicología/cultura y sus implicancias para repensar la situación política de la psicología. Contenido. Arte, Cultura y Ciencias Sociales(3), 113-121.

Ortiz, F., Vieyra, A. y Orozco, C. (2015). Narrativas sobre el lugar. Habitar una vivienda de interés social en la periferia urbana. Revista INVI, 30(84), 59-86. doi:10.4067/S0718-83582015000200003

Parker, I. (1996). Discurso, cultura y poder en la vida cotidiana Psicologías, discursos y poder: PDP. Madrid: Visor.

PNUD. (2012). Bienestar subjetivo: el desafío de repensar el desarrollo. Recuperado de http://desarrollohumano.cl/idh/informes/2012-bienestar-subjetivoel-desafio-de-repensar-el-desarrollo/

Patton, M. (2002). Qualitative research and evaluation methods. Thousand Oaks, CA: Sage.

Pol, E. (1996). La apropiación del espacio Cognición, representación y apropiación del espacio (pp. 4562). Barcelona: Publicacions de la Universitat de Barcelona.

Rodríguez, A. y Sugranyes, A. (2004). El problema de vivienda de los "con techo". EURE Revista Latinoamericana de Estudios Urbano Regionales, 30(91), 53-65. doi:10.4067/ S0250-71612004009100004 
Sabatini, F., Cáceres, G. y Cerda, J. (2001). Segregación residencial en las principales ciudades chilenas: Tendencias de las tres últimas décadas y posibles cursos de acción. EURE Revista Latinoamericana de Estudios Urbano Regionales, 27(82), 5-24. doi:10.4067/ S0250-71612001008200002

Sanín Santamaría, J. (2008). Hogar en tránsito. Apropiaciones domésticas de la vivienda de interés social (VIS) y reconfiguraciones del sentido de hogar. Antípoda, (7), 31-61.

Sisto, V. (2009). Transformaciones sociales, individualización y subjetividad. Una mirada desde Iberoamérica a los cambios que están marcando nuestro tiempo. Psicoperspectivas, 8(2), 1-11.

Skewes, J. C. (2005). De invasor a deudor: el éxodo desde los campamentos a las viviendas sociales en Chile. En A. Rodríguez y A. Sugranyes (Eds.), Los con techo. Un desafío para la política de vivienda social (2a ed., pp. 103-124). Santiago: SUR.

Starks, H. y Brown, S. (2007). Choose your method: a comparison of phenomenology, discourse analysis and grounded theory. Qualitative Health Research, 17(10), 1372-1380. doi:10.1177/1049732307307031

TECHO. (2013). Actualización del catastro nacional de campamentos. Informe de cifras generales. Santiago: Autor.

Touraine, A. (1994). Crítica de la modernidad. Buenos Aires: Fondo de Cultura Económica.
Vidal Moranta, T. y Pol Urrútia, E. (2005). La apropiación del espacio: una propuesta teórica para comprender la vinculación entre las personas y los lugares. Anuario de Psicología, 36(3), 281-297. 